\title{
The Unmet Need in Population Stablization
}

More than 100 million women in less developed countries, or about 17 percent of all married women, would prefer to avoid a pregnancy but are not using any form of family planning. Demographers refer to these women as having an "unmet need" for family planning. Unmet need for contraception can lead to unintended pregnancies, which pose risks for women, their families, and societies. A particularly dangerous consequence is an unsafe abortion, a leading cause for maternal mortality. Unwanted births pose risk for children's health and wellbeing and contribute to rapid population growth in resource-strapped countries. Due to these implications, the unmet need concept has influenced the development of family planning programs for more than 20 years. The concept of unmet need points to the gap between some women's reproductive intentions and their contraceptive behavior.

Today, the main tool for measuring unmet need is the Demographic and Health Survey (DHS), which has been conducted in 55 countries.[1] Married women aged 15 to 49 are interviewed regarding their future reproductive intentions. These responses are linked to the current contraceptive prevalence and practices to arrive at an estimate of the unmet need. In spite of some shortcomings such as exclusion of married women, DHS remains the benchmark tool for assessing the unmet need.

Generally speaking, as countries develop, fertility declines with increasing contraceptive. The unmet need in developed countries is low. In most European countries, the unmet need is less than $10 \%$. The unmet need may also be low in countries where the culture encourages women to have more children. For example, in Chad, though the average fertility is high (women have more than 6 children on average), the unmet need is only $4 \%$. Comparatively, countries where the desire for a large family is reducing, but the effective distribution of contraceptive technology has not occurred represent areas where the unmet need is high. Countries such as Haiti, Nepal, and Uganda have an unmet need over $30 \%$. India had a contraceptive prevalence rate of $48.3 \%$ in 2003 . The unmet need was estimated as $15.8 \%$. Of this, the unmet need for spacing births was $8.3 \%$ and for limiting births was $7.5 \%$.

Women have unmet needs for a variety of reasons and usually it is a combination of a number of factors which brings about this situation. The reasons are complex and amorphous, often intertwined with cultural and religious beliefs. Some of the recurring themes for not using contraception are:

- Perceived lack of exposure to pregnancy amongst couples who have intercourse infrequently or are breastfeeding or approaching menopause. These couples typically also have an ambivalent attitude towards becoming pregnant.

- Opposition to family planning methods from women, their husbands or other members of the family.

- Method related problems such as side-effects, cost and access facilities.

- Lack of knowledge of contraception.

Women who have an unmet need are typically on the lower rungs of the socioeconomic and education ladder. These women represent the unserved population in terms of many other indicators of health care in general. Women who have used contraceptives in the past and have a positive intent of using them again are the most likely to have their unmet need fulfilled. These are typically women who are most likely to use contraception in the near future and the easiest to serve. On 
the other extreme, are women who have never used contraception in the past and have no positive intention of using a contraceptive but still wish to avoid pregnancy. This population is the least likely to adopt contraceptive practices. They are the most difficult to reach. The proportion of women with this characteristic varies with different populations. 54\% of Indian women with unmet need fall into this category.

Survey data that is gathered from the DHS is an indicator and guideline for family planning programs. This data is a useful tool to identify the extent of the problem, the obstacles to reaching women and weaknesses in the service infrastructure. Family planning programs can take some important steps to reduce the barriers that women face in their efforts to obtain contraceptive methods and services:

- Women need to be counseled on the full range of available contraceptive methods so that they can choose the method that best matches their individual circumstances and intentions and can change methods when they need to.

- Women who are postpartum, breastfeeding, or approaching menopause need counseling on their likelihood of becoming pregnant and on what family planning methods might be appropriate for them.

- Women need correct information on contraceptive methods, especially on side effects and how to manage them.

- Programs should work to improve interpersonal relations between clients and providers and to ensure periodic follow-up of clients to reduce the number of women who stop using contraception.

- Programs should focus on men as well as women, creating an environment in which both sexes can seek services and encouraging men to discuss family planning with their wives.

India's population policy has resulted in the total fertility rate declining from 6 in the 1960s to 2.85 in 2003.
This is undoubtedly a success but only a partial one. The sheer size of the population requires us to redouble our efforts and ensure replacement level fertility at the earliest. In 2000 the contraceptive prevalence rate (CPR) among married women was $48.3 \%$. Contraceptive use in India is characterized by:

- The predominance of non-reversible methods, particularly female sterilization (about $35 \%$ of couples use female sterilization while only $3 \%$ of male partners are sterilized).

- Limited use of male-/couple-dependent methods.

- High discontinuation rates.

- Negligible use of contraceptives among both married and unmarried adolescents.

Less than $7 \%$ of married couples use officially accepted effective spacing methods (condoms, pills or intrauterine devices). [2] This represents the segment which needs the maximum promotion. Couples need to be educated and awareness needs to be spread about these methods. The results from the next DHS will be available in 2013 and should reflect changing patterns of contraceptive use.

\section{References}

1. Unmet need for family planning: Recent Trends and Their Implications for Programs. Population Research Bureau, 2003.

2. "Looking Back, Looking Forward: A profile of sexual and reproductive health in India." Population Council with support from World Health Organization. New Delhi, 2003. 